\title{
Micromagnetic modelling of ferromagnetic cones
}

\author{
Richard P. Boardman, Hans Fangohr, Matthew J. Fairman, Jürgen Zimmermann, and Simon J. Cox \\ Computational Engineering and Design Group \\ School of Engineering Sciences \\ University of Southampton \\ United Kingdom
}

Alexander A. Zhukov and Peter A. J. de Groot

School of Physics and Astronomy

University of Southampton

United Kingdom

(Dated: March 5, 2006)

\begin{abstract}
Using micromagnetic modeling, we calculate numerically the magnetization reversal of soft permalloy cones of height and diameter $\leq 100 \mathrm{~nm}$. By varying the cone height and the base diameter in steps of $10 \mathrm{~nm}$ and $5 \mathrm{~nm}$, respectively, we map the remanent states systematically. We observe reversal mechanisms with remanent configurations ranging from single domains at small diameters, through buckle states and complex C-states through to vortex states at larger diameters and heights. We present a phase-diagram of the remanent states of magnetization as a function of diameter and height. For the largest cones investigated, we find magnetization configurations in the reversal process which consist of two superimposed vortices with cores pointing in orthogonal directions.
\end{abstract}

\section{INTRODUCTION}

Requirements for magnetic applications are driving length scales deep into the sub-micron range. At such length scales the magnetic behavior of objects is strongly influenced by their shape. A number of two- and threedimensional shapes have been investigated experimentally and theoretically as candidates for novel patterned media, magnetic sensors and other nanomagnetic materials and devices. This includes cylinders and discs [1-4], spheres [5-7], part-spheres [8, 9], triangles [10] and other polygons [10-12].

Magnetic model systems with conical or truncatedconical shapes are the starting point for the study of various structures. This includes point contact devices such as magnetic force microscopes and spin polarized scanning tunneling microscopes. The huge magnetoresistance effect, observed in magnetic point contacts, continues to be a subject of much controversy [13, 14]. A detailed understanding of the possible magnetic structures in cones is also an important input into these investigations. Furthermore, many nanostructure arrays prepared by interference or e-beam lithography often consist of tapered pillars which are geometrically conical $[15,16]$.

The magnetization reversal in truncated $\mathrm{Ni}$ and $\mathrm{Co}$ cones with base diameters of $80-120 \mathrm{~nm}$ have been studied experimentally and compared to numerical studies of truncated square based pyramids [17].

In this paper, we study the magnetic reversal behavior of permalloy cones with base diameters ranging from $10 \mathrm{~nm}$ and $100 \mathrm{~nm}$ and the aspect ratios of height to base diameter varying from 0.1 and 10 . For each geometry, we compute the remanence magnetization and create a phase diagram as a function of diameter and height. Fi-
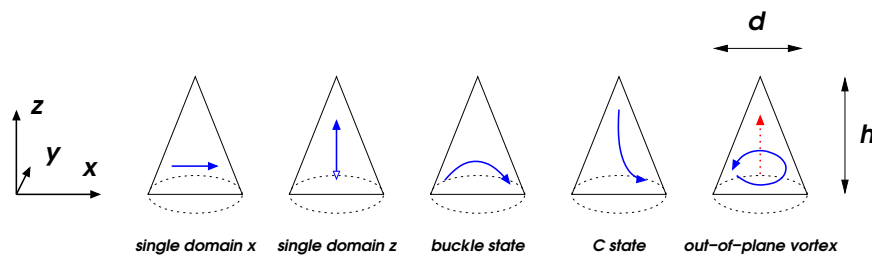

single domain $x$ single domain $z$

buckle state

Cstate

out-of-plane vortex

FIG. 1: Representation of remanent magnetization states; the solid arrows indicate the magnetization direction, the dotted arrow shows the vortex core direction. From left to right: single domain state in $x$, single domain state in $+z$ (filled arrow) and $-z$ (hollow arrow), buckle state [18], C state and out-ofplane vortex state (i.e. where the vortex core is perpendicular to the base).

nally, we present the hysteresis loop and magnetization reversal of the largest system in more detail.

\section{METHOD}

To compute the phase diagram we use the finite difference (FD) micromagnetic simulation program OOMMF [19] over clusters of PCs running Condor [20, 21] on Microsoft Windows. We have also used the hybrid finite element/boundary element (FE/BE) micromagnetic simulation program magpar [22] on machines running GNU/Linux. In both software packages we have used the Landau-Lifshitz Gilbert equation [23, 24] to compute metastable configurations.

We use the material parameters for $\mathrm{Ni}_{80} \mathrm{Fe}_{20}$ permalloy $\left(J_{s}=1.0 \mathrm{~T}, A=1.3 \times 10^{-11} \mathrm{~J} / \mathrm{m}, K_{1}=0.0 \mathrm{~J} / \mathrm{m}^{3}\right)[25]$ 
and a damping constant $\alpha$ of 0.25 to improve convergence. We perform simulations on cones where the overall diameter $d$ of the base ranges from $10 \mathrm{~nm}$ to $100 \mathrm{~nm}$, the height $h$ between the base and the tip ranges from $10 \mathrm{~nm}$ to $100 \mathrm{~nm}$ and the external magnetic field is applied along the $x$ direction of the cone (see figure 1 ). The mesh for the hybrid FE/BE simulation is created using NETGEN [26]. For the cone where $d=100 \mathrm{~nm}$ and $h=100 \mathrm{~nm}$, the mesh contains 210825 tetrahedra, with the largest element having an edge length smaller than $4 \mathrm{~nm}$, which is below [27] the calculated exchange length $\lambda_{\mathrm{ex}}=\sqrt{2 A / \mu_{0} M_{s}^{2}}[28]$ of $5.71 \mathrm{~nm}$.

The remanent magnetization configurations computed using OOMMF and magpar are in agreement providing the shape is sufficiently well resolved in both cases. To ensure this is the case with the FD method, the cones were discretized into 8000 cells, in the largest case the cell edge length is $5 \mathrm{~nm}$ with a maximum adjacent cell spin angle below 0.5 radians.

Starting from an initially uniform magnetization state pointing in the $+x$ direction, we apply a magnetic field of $500 \mathrm{mT}$ capable of maintaining a nearly homogeneous magnetization and reduce this field in steps of $1 \mathrm{mT}$ until the magnetization is reversed to compute the hysteresis loop. When the applied field is reduced to zero, we classify the magnetization pattern to create the remanence phase diagram. Figure 1 shows schematic plots of the observed remanent states.

\section{RESULTS}

Figure 2 shows the phase diagram for the remanent magnetization states in cones where $10 \mathrm{~nm} \leq d \leq 100 \mathrm{~nm}$ and $10 \mathrm{~nm} \leq h \leq 100 \mathrm{~nm}$ and the applied field was originally in the $+x$ direction (see figure 1 ). Where $h$ is less than 20nm, the remanent state is a single domain state with the magnetization pointing in the $x$ direction (figure 1 , left). If $h$ is above $80 \mathrm{~nm}$ and the ratio $h / d$ is high (i.e. a tall, thin cone) then the single domain state in $z$ is preferable due to shape anisotropy (figure 1, second from left). We have observed two types of single domain states in $z$ : one with the magnetization pointing up towards the tip of the cone and the other with the magnetization pointing down towards the base. These single domain states in $x$ and $z$ have been reported in experimental studies [17].

Moving to large $h$ and $d$ the demagnetizing energy grows and the remanent state is the vortex state with the magnetization in the core of the vortex pointing out of the $x y$ plane (figure 1, right).

For intermediate values of $h$ we find at large $d$ the buckle state (figure 1, centre), in which the overall magnetization points in $x$ but around the centre of the $x y$ plane this bends slightly upwards and downwards in $z$. The buckling is an indication of the growing dipolar en-

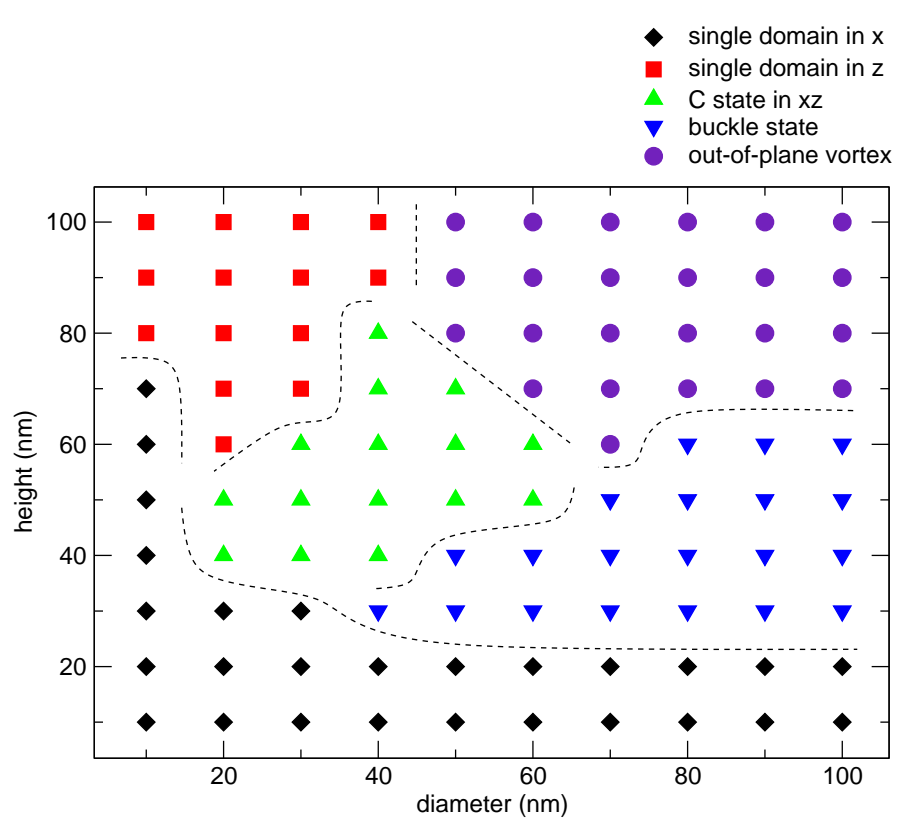

FIG. 2: Phase diagram of the remanent magnetization states for cones where the applied field was originally in the $+x$ direction. The symbols represent computed points, dashed lines are guides to the eye

ergy of the single-domain state.

At smaller $d$ for intermediate $h$ the remanent state is a C-shaped configuration (figure 1, second from right). The $\mathrm{C}$ state is related to the single domain state in $z$ by the shape anisotropy driving the magnetization to point primarily in the $-z$ direction. The $\mathrm{C}$ state reduces the demagnetization energy and the bending of the magnetization in the $+x$ direction close to the base of the cone shows the history of the system: prior to the field being reduced to zero the magnetization was pointing in the $+x$ direction.

In addition to the static remanent configurations presented above, we have studied the dynamics of the magnetization reversal for selected geometries. For the larger geometries with an out-of-plane vortex remanent configuration, we observe intermediate magnetization configurations in the hysteresis loop which exhibit two superimposed vortices with perpendicular orientation. Figure 3 shows the hysteresis loop with inset magnetization cross section snapshots, and figure 4 shows the superimposed vortices that occur within the hysteretic reversal process.

Figure 3 shows the complete hysteresis loop for a cone with $d=h=100 \mathrm{~nm}$. When the applied field is reduced from saturating the magnetization in the $+x$ direction, it forms an in-plane vortex (i.e. where the magnetization circulates in the $y z$ plane and the vortex core points in the $x$ direction) shown at point A. The same behavior is observed for the magnetization reversal of spheres $[6,9]$ of similar size. Note that this configuration is not observed in cones in the absence of an applied field and therefore 
this state is not shown in figure 1.

The in-plane vortex is replaced by an out-of-plane vortex (i.e. where the magnetization circulates in $x y$ and the vortex core points in the $z$ direction) shown at point $B$ after overcoming an energy barrier. The core of this vortex is anchored at the tip of the cone and compensates for the applied field by tilting the core, allowing the majority of the magnetization to align with the applied field, thus minimizing Zeeman energy.

A further reduction of the field (point C) causes the core of the vortex to shift to the centre of the cone. Reducing the field below zero causes the vortex to bend in the opposite direction to point B. At $B_{\mathrm{x}}=0 \mathrm{mT}$ the magnetization is in the vortex state as shown in figure 1 (right). Another energy barrier needs to be overcome to destroy the out-of-plane vortex, leaving the magnetization with an in-plane vortex (point D) with the core pointing in the opposite direction to the vortex at point A.

Once the field is sufficiently high the in-plane vortex aligns into a homogeneous saturated magnetization in the $-x$ direction for $\left|B_{\mathrm{x}}\right| \geq 250 \mathrm{mT}$.

Figure 4 shows specifically the magnetization at points E, F, G and H from figure 3 to explain the subtle "kinks" in the hysteresis loop. The top row shows a magnetization cross section along the height of the cone and the bottom row that along the base. The middle row is a schematic representation of the two vortices corresponding to the cross sections above and below.

Starting at point E, the system contains two vortices: the out-of-plane vortex with the core pointing in the $+z$ direction introduced at point $\mathrm{B}$, and the formation of an in-plane vortex with the core in the $-x$ direction parallel to the applied field. Increasing the field in the $-x$ direction causes the in-plane vortex to become more dominant (point F). A further increase of the applied field in the $-x$ direction allows the in-plane vortex to become even more influential, moving the out-of-plane vortex to the edge of the sample (point $\mathrm{G}$ ). There is a small energy barrier present to force the out-of-plane vortex from the system; once this has been overcome only the in-plane vortex remains (point $\mathrm{H}$ ).

\section{SUMMARY}

We have simulated the magnetization reversal in conical samples, and five separate remanent states - singledomain in $x$, single-domain in $z$, out-of-plane vortex, buckle and C-state - have been observed.

By analysing the remanent magnetization configuration for a series of simulations, we have created a phase diagram showing these results. The dynamics of the magnetization reversal are more complicated to classify and describe.

We have investigated and presented in more detail the

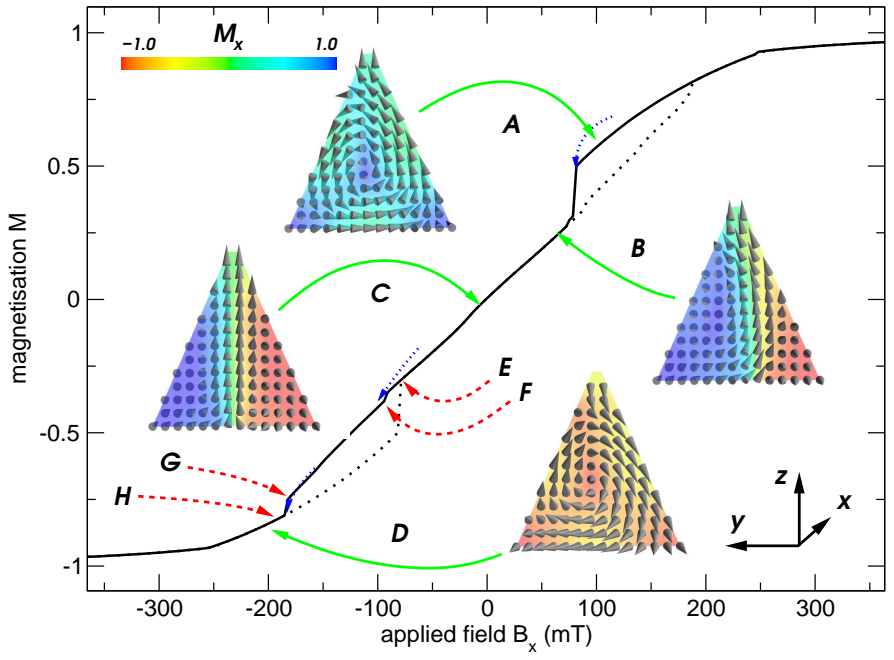

FIG. 3: Hysteresis loop and reversal mechanism for a cone where $d=h=100 \mathrm{~nm}$ and the applied field is across the diameter. Points E, F, G and H are explained in figure 4.

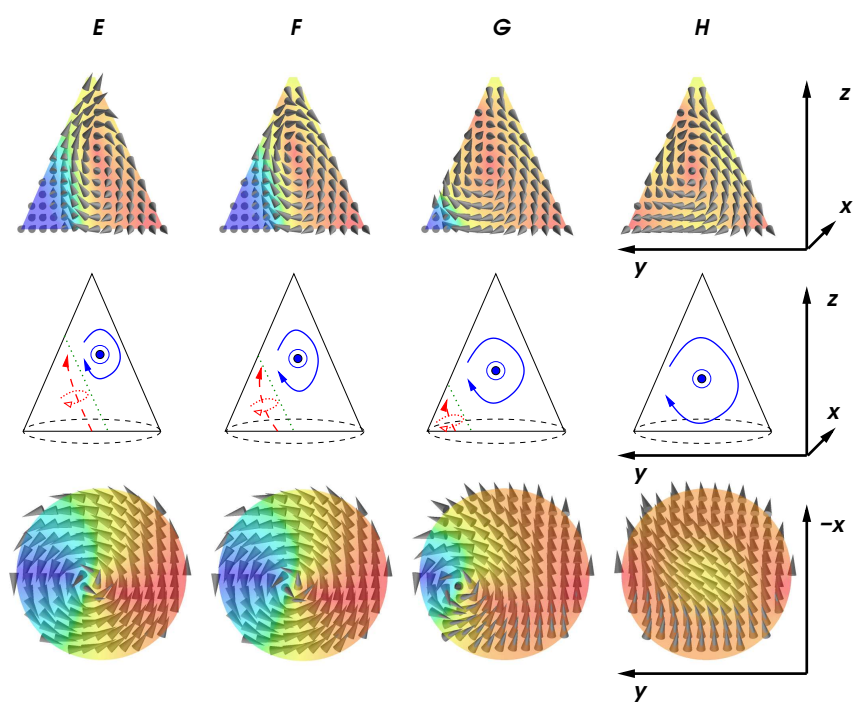

FIG. 4: Reversal mechanism detail for points E, F, G and H in figure 3. Top: $y z$ cutplanes of magnetization shaded by the $x$ component of the magnetization $\left(M_{x}\right)$. Middle: schematics indicating vortex behavior (solid arrows for the in-plane vortex shown the in top row, dashed arrows for the out-of-plane vortex shown in the bottom row). Bottom: $x y$ cutplanes of the magnetization at the base of the cone.

magnetization reversal of larger cones (height greater than $70 \mathrm{~nm}$ and diameter greater than $50 \mathrm{~nm}$ ) and find that for parts of the hysteresis loop, two coexisting vortices can be found in the magnetization (with cores pointing in approximately perpendicular directions). 
[1] R. P. Cowburn, A. O. Adeyeye, and M. E. Welland, New Journal of Physics 1, 16 (1999).

[2] R. P. Cowburn, D. K. Koltsov, A. O. Adeyeye, M. E. Welland, and D. M. Tricker, Phys. Rev. Lett. 83, 1042 (1999).

[3] S. P. Li, M. Natali, A. Lebib, A. Péin, Y. Chen, and Y. B. $\mathrm{Xu}$, Journal of Magnetism and Magnetic Materials 241, 447 (2002).

[4] J. K. Ha, R. Hertel, and J. Kirschner, Phys. Rev. B 67, 064418 (2003)

[5] J. C. H. Stapper, J. Appl. Phys. 40, 798 (1969).

[6] I. Eisenstein and A. Aharoni, J. Appl. Phys. 47, 321 (1975).

[7] J. Lam, J. Appl. Phys. 72, 5792 (1992).

[8] R. P. Boardman, H. Fangohr, A. V. Goncharov, A. A. Zhukov, P. A. J. de Groot, and S. J. Cox, J. Appl. Phys. 95, 7037 (2004).

[9] R. P. Boardman, J. Zimmermann, H. Fangohr, A. A. Zhukov, and P. A. J. de Groot, J. Appl. Phys. 97, 10E305 (2005).

[10] R. P. Cowburn, Journal of Physics D: Applied Physics 33, R1 (2000).

[11] R. P. Cowburn and M. E. Welland, Phys. Rev. B 58, 9217 (1998).

[12] M. Hwang, M. Redjdal, F. B. Humphrey, and C. A. Ross, J. Appl. Phys. 89, 7582 (2001).

[13] N. García, M. Muñoz, and Y.-W. Zhao, Phys. Rev. Lett. 82, 2923 (1999).

[14] J.-E. Wegrowe, T. Wade, X. Hoffer, L. Gravier, J.-M. Bonard, and J.-P. Ansermet, Phys. Rev. B 67 (2003).
[15] S. Y. Chou, P. R. Krauss, and L. Kong, J. Appl. Phys. 79, 6101 (1996)

[16] S. Y. Chou, Proceedings of the IEEE 85, 652 (1997).

[17] C. A. Ross, M. Farhoud, M. Hwang, H. I. Smith, M. Redjdal, and F. B. Humphrey, J. Appl. Phys. 89, 1310 (2001).

[18] D. K. Koltsov, R. P. Cowburn, and M. E. Welland, J. Appl. Phys. 88, 5315 (2000).

[19] M. J. Donahue and D. G. Porter, OOMMF User's Guide, National Institute of Standards and Technology, Gaithersburg, MD (1999), interagency Report NISTIR 6376.

[20] M. Litzkow, in Usenix Summer Conference (1987), pp. 381-384.

[21] M. Litzkow, M. Livny, and M. Mutka, in 8th International Conference of Distributed Computing Systems (1988), pp. 104-111.

[22] W. Scholz, J. Fidler, T. Schrefl, D. Süß, R. Dittrich, H. Forster, and V. Tsiantos, Computational Materials Science 28, 366 (2003).

[23] L. D. Landau and E. M. Lifshitz, Physikalische Zeitschrift der Sowjetunion 8, 153 (1935).

[24] T. L. Gilbert, Physical Review 100, 1243 (1955).

[25] R. Skomski and J. M. D. Coey, Permanent Magnetism, Series in Condensed Matter Physics (Institute of Physics, 1999).

[26] J. Schöberl, Netgen 4.3 User's Guide, http://www.hpfem.jku.at/netgen/ng4.pdf (2003).

[27] M. J. Donahue and R. D. McMichael, Physica B 233, 272 (1997)

[28] H. Kronmüller and M. Fähnle, Micromagnetism and the Microstructure of Ferromagnetic Solids (Cambridge University Press, 2003), 1st ed. 